\title{
Blood flow reprograms lymphatic vessels to blood vessels
}

\author{
Chiu-Yu Chen, ${ }^{1}$ Cara Bertozzi, ${ }^{1}$ Zhiying Zou, ${ }^{1}$ Lijun Yuan, ${ }^{1}$ John S. Lee, ${ }^{1}$ MinMin Lu, ${ }^{1}$ \\ Stan J. Stachelek, ${ }^{2}$ Sathish Srinivasan, ${ }^{3}$ Lili Guo, ${ }^{1}$ Andres Vincente, ${ }^{4}$ Patricia Mericko, ${ }^{1}$ \\ Robert J. Levy, ${ }^{2}$ Taija Makinen, ${ }^{4}$ Guillermo Oliver, ${ }^{3}$ and Mark L. Kahn ${ }^{1}$ \\ 1Department of Medicine and Division of Cardiology, University of Pennsylvania, Philadelphia, Pennsylvania, USA. Department of Cardiology, \\ The Children's Hospital of Philadelphia, Philadelphia, Pennsylvania, USA. ${ }^{3}$ Department of Genetics, St. Jude Children's Research Hospital, \\ Memphis, Tennessee, USA. ${ }^{4}$ Lymphatic Development Laboratory, Cancer Research UK, London, United Kingdom.
}

\begin{abstract}
Human vascular malformations cause disease as a result of changes in blood flow and vascular hemodynamic forces. Although the genetic mutations that underlie the formation of many human vascular malformations are known, the extent to which abnormal blood flow can subsequently influence the vascular genetic program and natural history is not. Loss of the SH2 domain-containing leukocyte protein of $76 \mathrm{kDa}$ (SLP76) resulted in a vascular malformation that directed blood flow through mesenteric lymphatic vessels after birth in mice. Mesenteric vessels in the position of the congenital lymphatic in mature Slp76-null mice lacked lymphatic identity and expressed a marker of blood vessel identity. Genetic lineage tracing demonstrated that this change in vessel identity was the result of lymphatic endothelial cell reprogramming rather than replacement by blood endothelial cells. Exposure of lymphatic vessels to blood in the absence of significant flow did not alter vessel identity in vivo, but lymphatic endothelial cells exposed to similar levels of shear stress ex vivo rapidly lost expression of PROX1, a lymphatic fate-specifying transcription factor. These findings reveal that blood flow can convert lymphatic vessels to blood vessels, demonstrating that hemodynamic forces may reprogram endothelial and vessel identity in cardiovascular diseases associated with abnormal flow.
\end{abstract}

\section{Introduction}

Human vascular malformations are common congenital diseases that can result in a variety of clinical disorders later in life. Patients may present with stroke and neurologic impairment from lesions in the central nervous system (1), high-output heart failure from arterio-venous shunting through large malformations (2), and disfigurement due to large or numerous cutaneous lesions (3). In the past two decades, significant progress has been made in identifying the genetic basis for many common human vascular malformations (4). These studies have revealed defects in many of the pathways known to regulate endothelial and vessel identity (e.g., NOTCH signaling in Alagille syndrome, ref. 5; TGF- $\beta$ signaling in hereditary hemorrhagic telangiectasia, ref. 6) and function (e.g., endothelial junction formation in cerebral cavernous malformation, ref. 7), and some human vascular malformations have been successfully modeled in genetically altered mice deficient in the same pathways $(8,9)$.

Despite progress in determining the genetic origins of human vascular malformation, the treatment of these lesions remains primarily mechanical and is limited by a lack of molecular understanding of the natural history of vascular malformations. A hallmark of human vascular malformations, especially those with significant clinical consequences, is the shunting of blood away from natural, hierarchical vascular circuits consisting of arteries, arterioles, capillaries, venules, and veins and into abnormal circuits that lack the organization and mechanisms normally used to control blood flow and hemodynamic forces. In the case of

Authorship note: Chiu-Yu Chen, Cara Bertozzi, and Zhiying Zou contributed equally to this work.

Conflict of interest: The authors have declared that no conflict of interest exists.

Citation for this article: J Clin Invest. 2012;122(6):2006-2017. doi:10.1172/JCI57513. large vascular malformations, such shunts frequently result in hemorrhage or high-output heart failure many years after they first form. Understanding whether and to what extent hemodynamic forces shape the molecular and genetic landscape of these vascular structures would provide much needed insight into how the pathologic syndromes associated with human vascular malformations arise and may be treated.

Mice lacking the SLP7 6 adaptor protein experience abnormal lymphatic vascular development, in which blood-lymphatic vascular connections form during embryonic life $(10,11)$. A fraction of such mice survive to adulthood, at which time they exhibit large arterio-venous malformations that form as a consequence of these connections (10). In contrast to experimental studies of hemodynamic effects on vessel identity and function that utilize surgical interventions, alterations in blood flow in $S l p 76^{--}$animals arise gradually as a consequence of vascular remodeling in much the same way as in human vascular malformations. In the present study, we determined the effect of blood flow and hemodynamic forces on the identity of the lymphatic vessels that constitute part of the efferent loop of the vascular malformation in $S l p 76^{-/-}$animals. Our studies demonstrate that lymphatic endothelial and vessel identity are negatively regulated by blood flow and that changes in hemodynamic forces can completely reprogram vessel identity in postnatal life. These findings suggest that the genetic basis for human vascular malformations is only the first half of a story that is also written by the molecular and genetic responses to hemodynamic forces.

\section{Results}

SLP76-deficient mice undergo late separation of the blood and lymphatic circulation. The hematopoietic signaling proteins SLP76 and SYK have been shown to regulate blood and lymphatic vascular sepa- 
ration during development (10). Animals lacking these proteins develop mixing of blood and lymphatic circulation in the skin and intestine, defects that result in embryonic edema and neonatal chylous ascites $(10,12,13)$. Despite these defects, approximately one-third of SLP76-deficient mice survive to adulthood, when they no longer appear edematous but instead exhibit a highcardiac-output state due to the presence of an intestinal vascular shunt (discussed below and in ref. 10). These findings suggested that the blood-lymphatic connections that form in SLP76-deficient animals might be transient and that vascular remodeling might result in both the resolution of blood-lymphatic mixing and formation of the vascular shunt. To better understand the natural history of the vascular defects in these animals, we analyzed the blood and lymphatic vessels in the skin and intestine of deficient animals and wild-type littermates at various time points during development and postnatal life.

Between E12 and E15, lymphatic vessels invade the skin as they grow by angiogenic sprouting from the central lymph sacs adjacent to the cardinal veins $(14,15)$. At this time point, $\operatorname{Sl} 76^{-1-}$ embryos were identifiable by marked cutaneous edema and the presence of blood-filled cutaneous lymphatics (Figure 1A). By E18, however, Slp $76^{-/-}$animals exhibited normal abdominal skin folds, indicating resolution of the edema, and blood-filled lymphatics appeared less visible or completely resolved (Figure 1A). Resolution of blood-filled lymphatics in the skin of late-gestation $S / p 76^{-1-}$ animals was also detectable histologically by the absence of red blood cells in cutaneous LYVE1+ lymphatic vessels (Figure 1B). A similar rapid resolution of the cutaneous vascular phenotype has also been reported for SYK-deficient embryos between E16.5 and E18.5, although the basis for this observation was not investigated in detail (12). Lymphatic vessels first appear in the mouse intestine after E15, a time point considerably later than their appearance in the skin (16). Significantly, in SLP76-deficient mice, blood-filled intestinal lymphatics and intestinal edema were observed after E18, a time point at which the skin phenotype was noted to have resolved (Figure 1B).

These findings suggested that blood-lymphatic connections in SLP76-deficient mice are transient and resolve at one site even as they form at another site in the same animal. To map the formation and resolution of connections between the blood and lymphatic circulations of these animals, we injected biotin-conjugated lectin into the blood to label endothelial cells in contact with circulating blood. Subsequent staining with FITC-streptavidin and antiLYVE1 antibodies was used to functionally identify lymphatic endothelium exposed to circulating blood. LYVE1- blood vessels but not LYVE1 $1^{+}$lymphatic vessels in the skin, intestine, and mesentery of wild-type neonates were positive for lectin staining (Figure 1C), confirming that circulating blood does not normally come into contact with lymphatic vessels. In contrast, in neonatal SLP76deficient animals, the LYVE1 $1^{+}$lymphatic vessels of the intestinal wall and mesentery were positive for lectin staining even though LYVE1 ${ }^{+}$lymphatic vessels in the skin were lectin negative (Figure 1C). Thus, blood-lymphatic mixing was present in the intestine but not the skin of neonatal SLP76-deficient mice. When blood-lymphatic mixing was studied using this approach in mature, 12-weekold littermates, however, the LYVE1+ ${ }^{+}$intestinal vessels of SLP76deficient mice were no longer lectin ${ }^{+}$(Figure 1D). These findings demonstrate that blood-lymphatic connections in SLP76-deficient mice ultimately resolve and suggest that the intestinal shunts previously described in mature animals form as a consequence of the vascular remodeling that resolves those connections.
Mesenteric vessels in the anatomic position of lymphatics lack lymphatic identity after exposure to blood flow in Slp $76^{-1-}$ mice. Mature SLP76deficient mice develop a high-cardiac-output state due to the presence of intestinal shunts in which afferent arterial blood is directly returned to the heart through efferent mesenteric vessels, bypassing the intestinal microvasculature (10). Previous angiographic studies have shown that the efferent shunt vessels (SVs) correspond anatomically to the mesenteric veins and collecting lymphatic vessels (10), suggesting that mesenteric lymphatics become annexed to the blood vascular system during the remodeling process that achieves vascular separation in the intestine. The results of the studies of blood-lymphatic mixing shown in Figure 1 were notable for indicating an absence of large LYVE1 $1^{+}$lymphatic vessels in the mesentery of mature SLP76-deficient mice, even though these animals had characteristic mesenteric vascular bundles containing a thick-walled artery and two thin-walled vessels consistent with the congenital vein and lymphatic vessels (Figure 1D). This finding suggested that the mesenteric lymphatic vessels exposed to flowing blood due to blood-lymphatic vascular mixing in the intestine of SLP76-deficient animals lose lymphatic identity.

To better define the identity of the endothelial cells lining the efferent SVs, we examined expression of prospero homeobox 1 (PROX1), a transcription factor that specifies and maintains lymphatic endothelial identity $(17,18)$; podoplanin (PDPN), a glycoprotein expressed by lymphatic but not blood endothelial cells $(19,20)$; the lymphatic marker LYVE1 (21); and vWF, a procoagulant protein synthesized by blood but not by lymphatic endothelial cells (LECs) (22, $23)$; in addition to the pan-endothelial protein PECAM. Mesentery from wild-type and SLP76-deficient neonates and wild-type 12-weekold animals contained thick-walled, PECAM1 ${ }^{+} \mathrm{vWF}{ }^{+} \mathrm{PROX} 1$ LYVE1- arteries, thin-walled PECAM1 ${ }^{+}{ }^{-} W F^{+} P R O X 1^{-}{ }^{-L Y V E 1}{ }^{-}$veins, and thin-walled PECAM $1^{+} \mathrm{PROX} 1^{+} \mathrm{PDPN}^{+} \mathrm{LYVE}^{+}{ }^{+} \mathrm{VWF}{ }^{-}$lymphatic vessels (Figure 2, A and B). At age 12 weeks, the artery and vein were visible as two adjacent blood-filled vessels in the mesentery of wild-type mature animals. In contrast, the mesentery of 12-week-old SLP76-deficient mice contained a third bloodcontaining vessel in the anatomic position of the congenital lymphatic that participates in the intestinal shunt (Figure 2B and see below; and ref. 10). These vascular bundles contained thick-walled, PECAM $1^{+} \mathrm{VWF}^{+} \mathrm{PROX} 1^{-} \mathrm{PDPN}^{-}{ }^{-} \mathrm{HVE} 1^{-}$arteries and large, thinwalled PECAM1 ${ }^{+} \mathrm{WWF}^{+}$PROX1-PDPN-LYVE1- SVs (Figure 2B).

Previous studies have shown that SLP76 is required in platelets to prevent the formation of blood-lymphatic connections in the intestine (10) and that these connections send blood flow through mesenteric collecting lymphatic vessels that ultimately carry efferent blood from a vascular shunt that forms in the intestine proper (11). To test whether the observed loss of lymphatic mesenteric collecting vessels in SLP76-deficient animals could result from loss of SLP76 in endothelial cells themselves, we next assessed the natural history of these vessels in Vav-Cre;Slp $76^{f l-}$ mice. Previous lineage tracing experiments performed using both Vav-Cre;R26RYFP and Vav-Cre;R26RYFP;Slp76fl- mice have revealed high-level Cre-mediated recombination in hematopoietic cells, with no detectable recombination in either blood or LECs of the intestine or elsewhere $(11,24)$. Mature Vav-Cre;Slp $76^{f l-}$ mice developed vascular shunts identical to those described in SLP76-deficient animals, and these vessels lacked lymphatic identity (Figure 3A). Thus, the loss of endothelial and vessel identity in mesenteric collecting lymphatics is a secondary event and not due to endothelial cellautonomous loss of SLP76 signaling. 
A

$\frac{n}{4}$

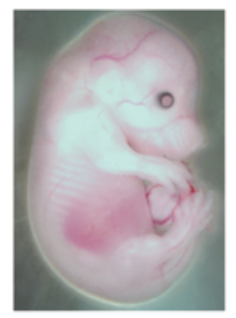

SIp76 6

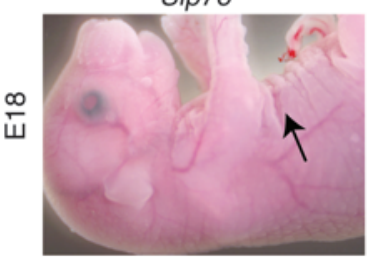

SIp $76^{-\alpha}$
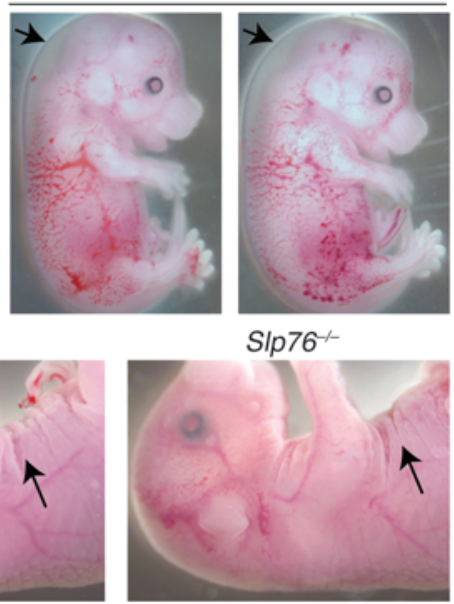

SIp76

C
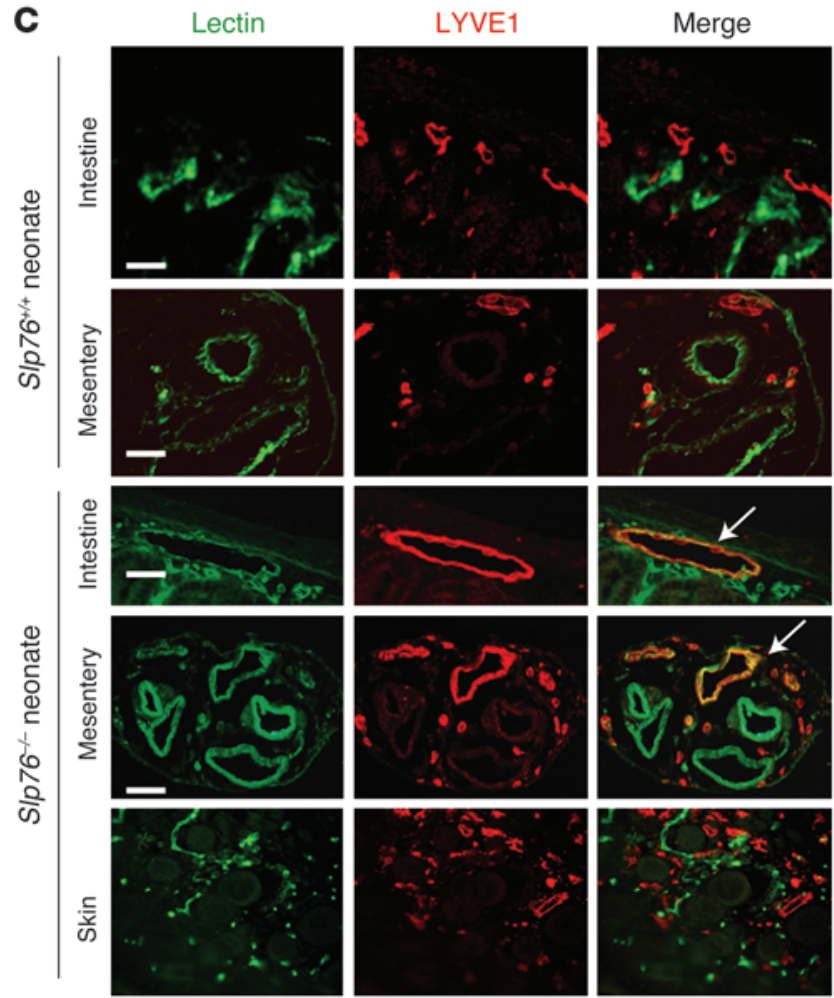

B

Skin
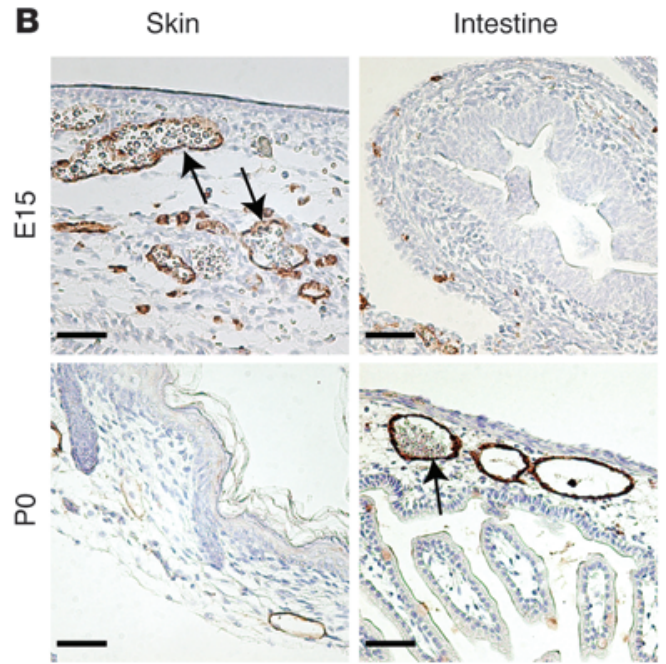

D
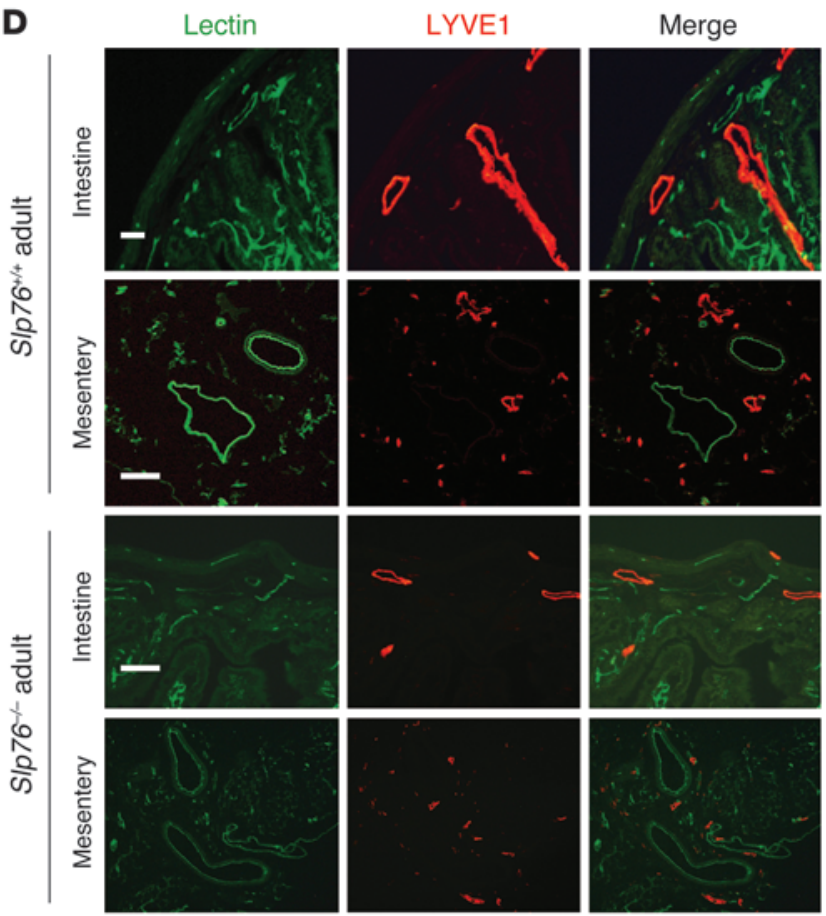

\section{Figure 1}

Late separation of the blood and lymphatic circulations in SLP76-deficient mice. (A) Cutaneous edema resolves in S/p76 ${ }^{-/-}$embryos between E15 and E18. Arrows indicate sites of raised skin indicative of edema at E15 (top) and the presence of normal skin folds indicating a lack of edema at E18 (bottom). (B) Blood-filled lymphatics resolve in the skin of SIp76 ${ }^{-1-}$ embryos at the same time that they appear in the intestine. LYVE1 staining (brown) identifies lymphatic vessels in the skin and intestine of E15 and neonatal S/p76-1- animals. Arrows indicate blood-filled lymphatics in E15 skin and in neonatal intestine. (C) Functional identification of blood-filled lymphatics in neonatal S/p76 ${ }^{-/-}$animals using intravenous injection of biotinylated lectin. Blood-perfused vessels are identified by FITC-streptavidin binding to biotin-lectin after intravenous injection (green), and lymphatic vessels are visualized by LYVE1 immunostaining (red). Co-stained vessels are present in the intestine and mesentery but not skin of SIp76-1- neonates (arrows). (D) Late resolution of mesenteric and intestinal blood-lymphatic mixing in SIp76 ${ }^{-1-}$ mice demonstrated using staining for injected biotin-lectin and LYVE1. Scale bars: $50 \mu \mathrm{m}$.

Finally, to determine whether the SVs that arise in $\operatorname{Slp} 76^{-1-}$ and $\mathrm{Vav}$-Cre;Slp $76^{f l-}$ mice acquire arterial or venous identity, we stained for EPHB4, a venous marker, and CX40, an arterial marker. SV endothelium was EPHB4+ and $\mathrm{CX}^{+} 40^{-}$, while that of mesenteric arteries was EPHB4- and $\mathrm{CX}^{-} 40^{+}$, a result consistent with venous identity (Figure 3A). These findings suggested that the mesenteric lymphatics of SLP76-deficient animals lose lymphatic vessel identity and acquire venous identity as they 
A
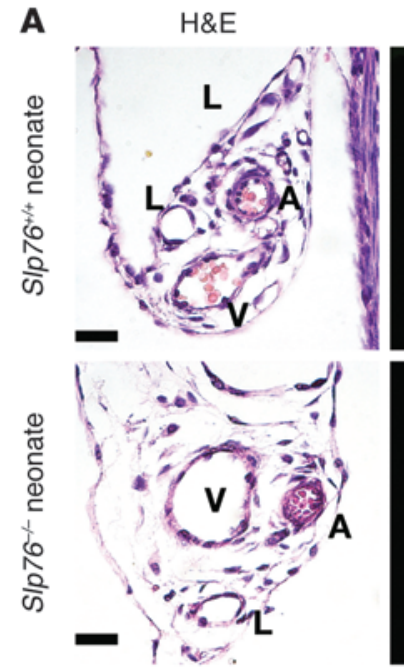

PECAM
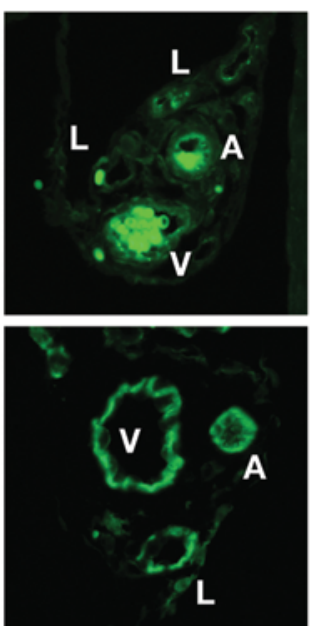

LYVE1/PROX1
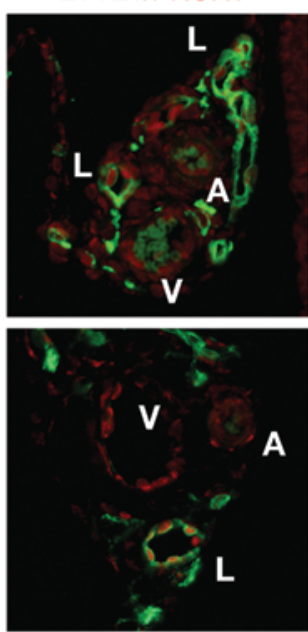

vWF
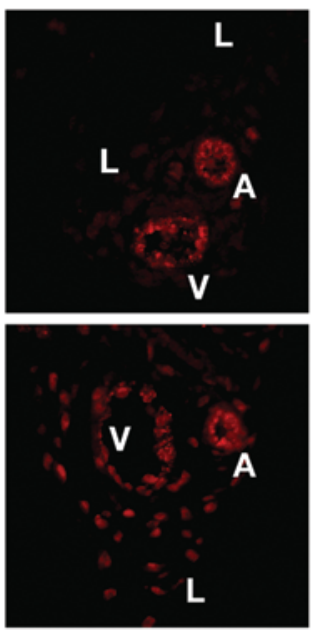

B

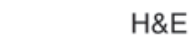

H\&E

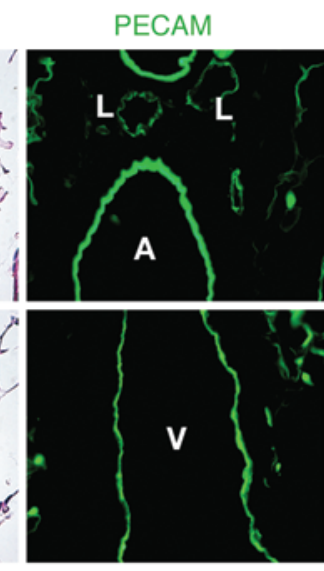

LYVE1/PROX1
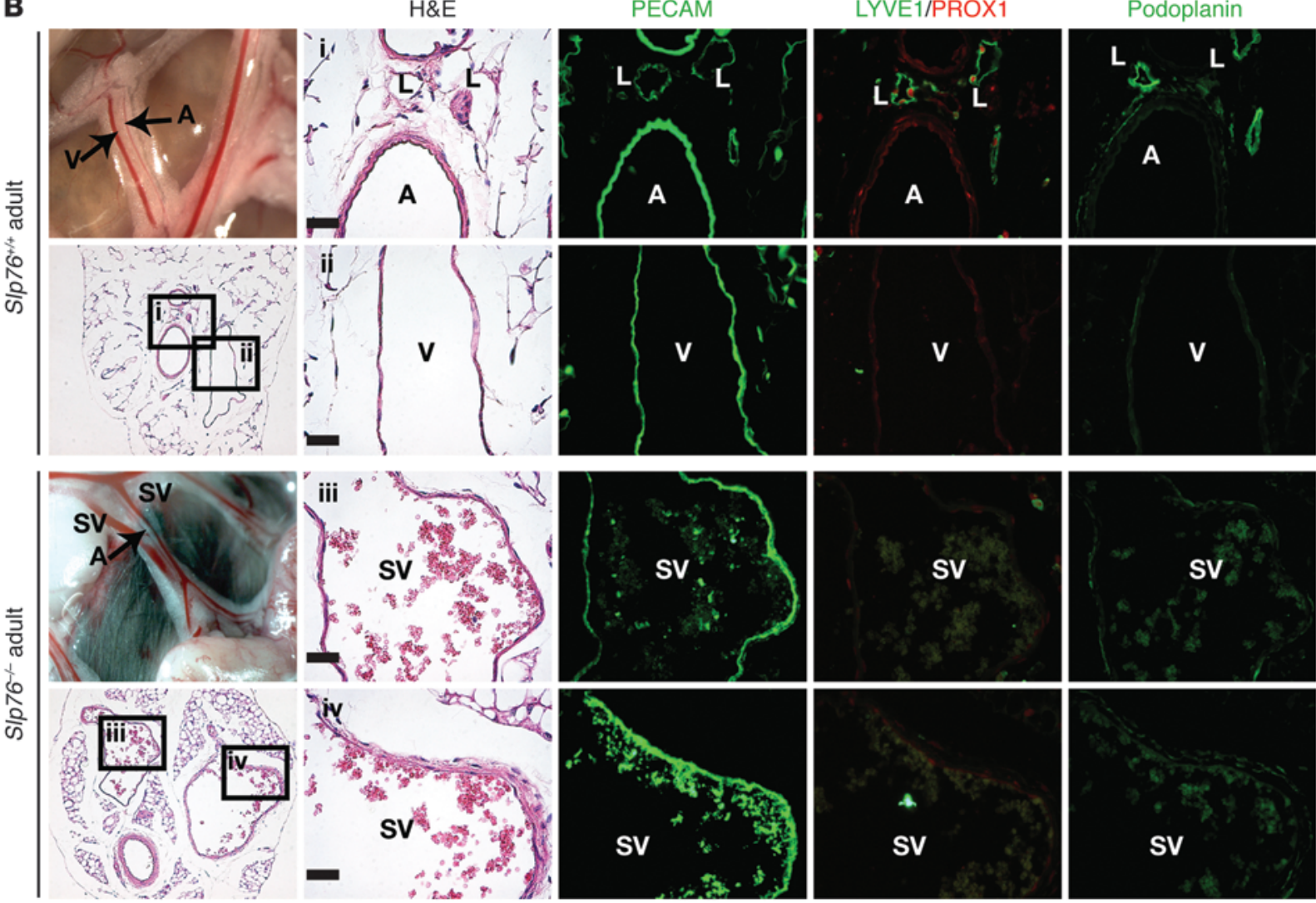

\section{Figure 2}

Mesenteric SVs in adult SIp76-/- mice lose lymphatic identity. (A) Neonatal mesenteric lymphatics in S/p76-/- mice express the lymphatic endothelial molecular markers LYVE1 and PROX1, but not the blood endothelial marker vWF. Shown is antibody staining of serial sections. (B) The mesenteric SVs in adult SIp76 ${ }^{-/-}$mice express PECAM but not LYVE1, PDPN, or PROX1. A, artery; V, vein; L, lymphatic. Scale bars: 20 um.

become exposed to blood flow from blood-lymphatic connections in the intestine (Figure 3B).

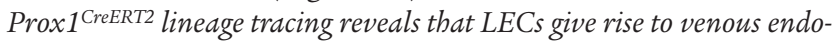
thelial cells in the mesenteric vessels of Slp $76^{-/-}$mice. The hypothesis that mesenteric lymphatic vessels in SLP76-deficient mice alter identity in response to blood flow is based on their characteristic anatomical position within the mesenteric vascular bundle. It is possible, however, that SVs do not derive from the congenital lymphatic due to a complex remodeling process. In addition, even if correctly identified, the endothelial cells lining this vessel 

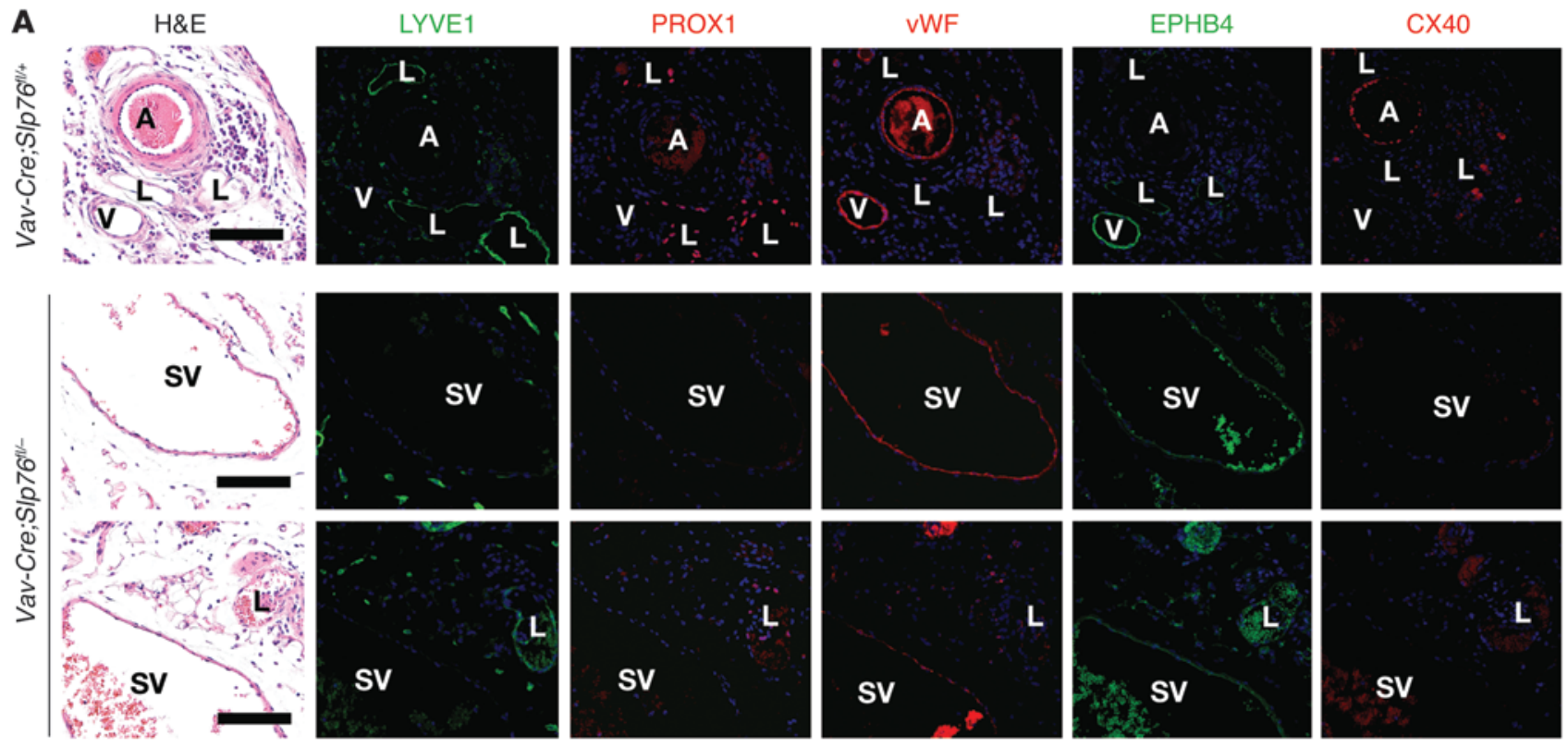

B
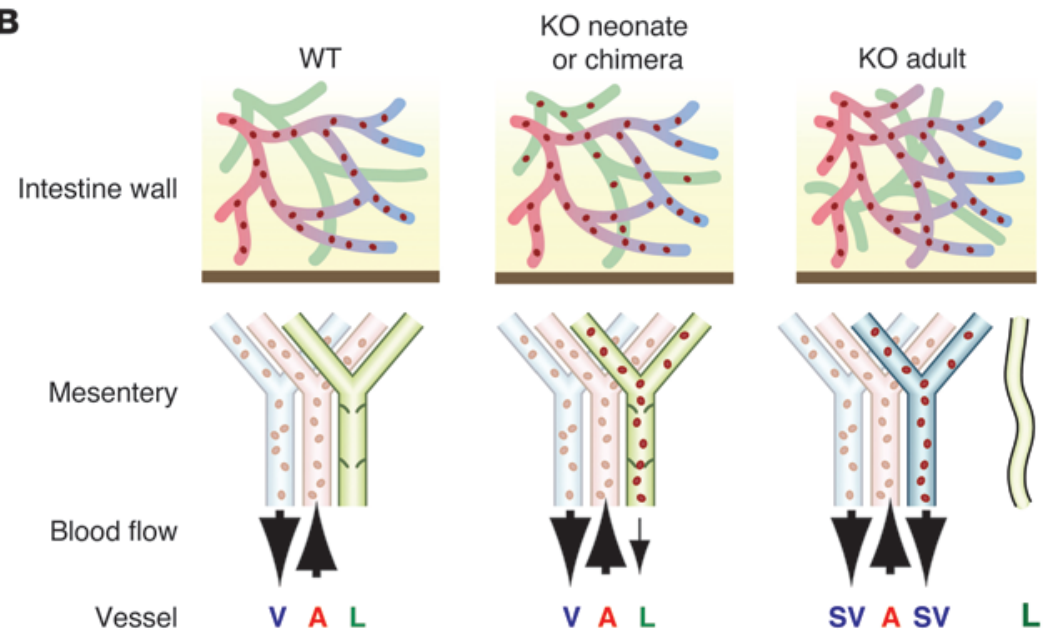

Figure 3

Mesenteric SVs in adult Vav-Cre;SIp76 fll- mice lose lymphatic identity and acquire venous identity. (A) Mesenteric SVs that form in Vav-Cre;SIp76 fllmice lose expression of lymphatic molecular markers and acquire expression of the blood vessel marker vWF and venous marker EPHB4 but not the arterial marker CX40. Scale bars: $50 \mu \mathrm{m}$. (B) Model of vascular remodeling in SLP76-deficient mice. Shown are the vascular anatomy and flow through the intestinal and mesenteric vessels of neonatal wild-type, neonatal S/p76-/- (KO neonate), and mature S/p76 ${ }^{-/-}$(KO adult) animals. SLP76-deficient radiation chimeras (KO chimera) develop a vascular phenotype like that observed in KO neonates. In the wild-type animal, afferent mesenteric blood flow is carried by the mesenteric artery ( $\mathrm{A}$, red), while efferent blood and lymph are carried by the mesenteric vein ( $\mathrm{V}$, blue) and lymphatic (L, green), respectively. In the KO neonate or KO chimera, blood-lymphatic mixing allows blood to enter the mesenteric lymphatics, but flow is minimal, and lymphatic identity is preserved. In the KO adult, mesenteric lymphatics become incorporated into an arterio-venous shunt that directs efferent blood flow through them (right), a process that produces an SV (blue) with blood vessel identity. Histologic studies reveal the presence of small lymphatic vessels that retain lymphatic identity in mature KO animals.

may have been molecularly reprogrammed or replaced to confer blood vessel identity. To determine whether LECs give rise to the blood endothelial cells that line efferent SVs, we bred SLP76deficient mice onto a Prox $1^{\text {CreERT2}}$;Rosa26RYFP background in which tamoxifen induction of CRE activity in Prox1-expressing cells activates permanent expression of YFP in LECs (15). Slp $76^{-/-}$;

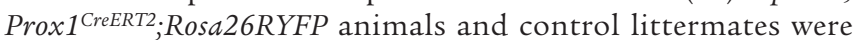
treated with tamoxifen from P1 to P5, a time point prior to intestinal vascular separation and the appearance of the intestinal shunt, and YFP was detected by immunohistochemistry at 12 weeks of age. Neonatal tamoxifen induction conferred mosaic YFP expression in the endothelial cells of lymphatic but not blood vessels in the mesentery of 12 week-old Slp $76^{+/-}$;Prox $1^{\text {CreERT2 }}$;Rosa26RYFP control animals (Figure 4A). $\mathrm{YFP}^{+}$endothelial cells were identified in the large mesenteric SVs that lack lymphatic identity in $S l p 76^{-1-}$;

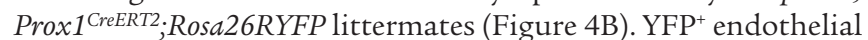
cells in these vessels were negative for PROX1, PDPN, and LYVE1 but expressed vWF (Figure 4B). These results suggested that 

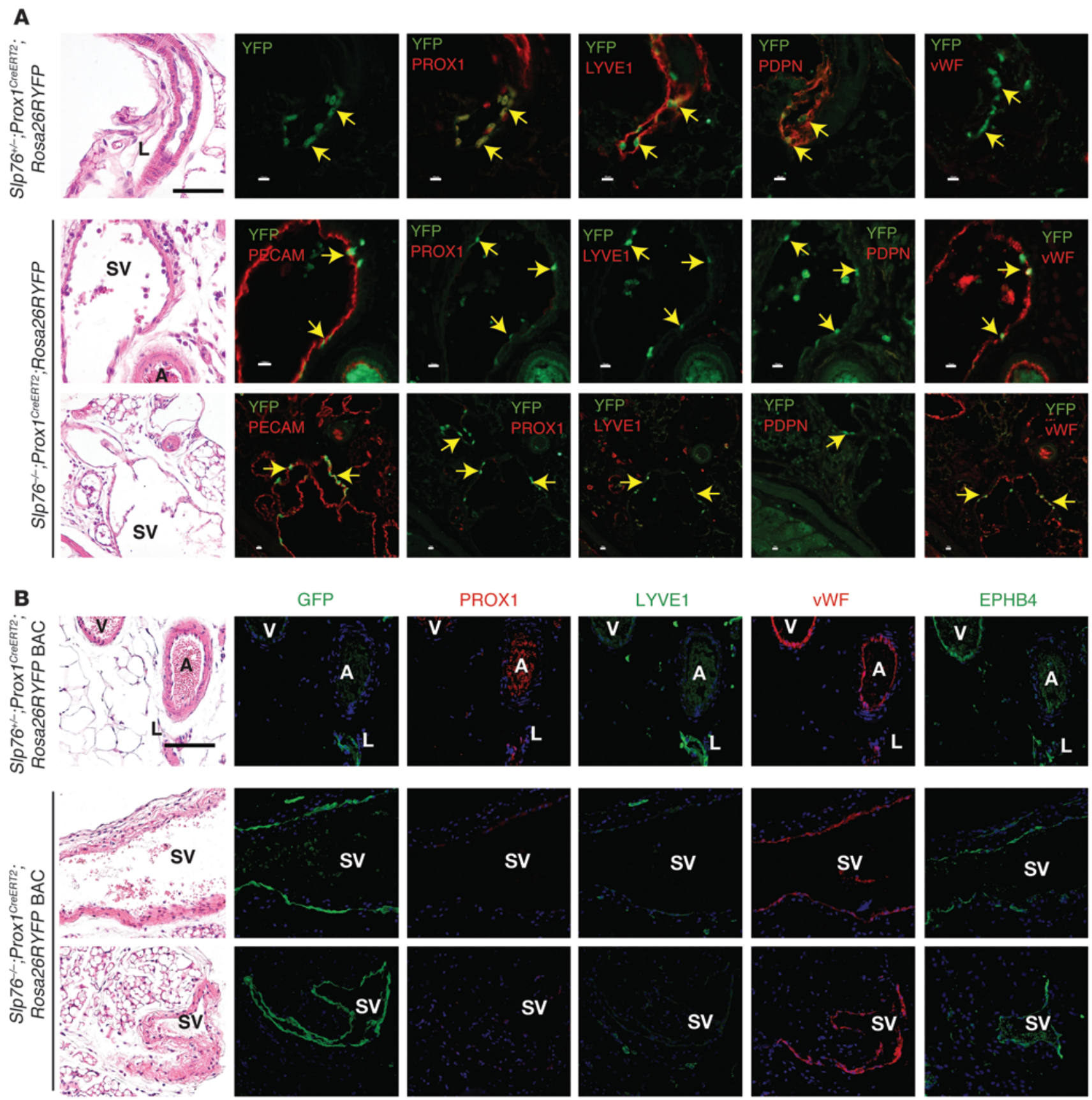

Figure 4

Genetic lineage tracing demonstrates that the blood endothelial cells lining mesenteric SVs in S/p76 ${ }^{-/-}$mice arise from LECs. (A) Lineage tracing

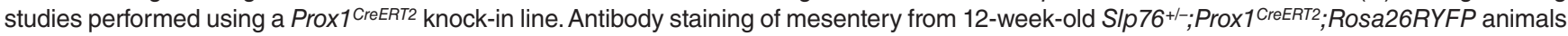
exposed to tamoxifen as neonates reveals YFP only in PROX1+LYVE1+PDPN+vWF-LECs (top). Antibody staining of mesentery from 12-week-old

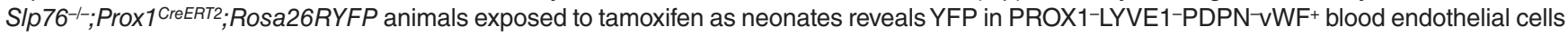
that line large SVs (bottom). Black scale bars: $50 \mu \mathrm{m}$; white scale bars: $10 \mu \mathrm{m}$. (B) Lineage tracing studies using a Prox 1 CreERT2 BAC transgenic line. Studies were performed as described in $\mathbf{A}$ using a single tamoxifen injection at P14. Note that with this Prox 1 CreERT2 line, virtually all the endothelial cells of the SVs shown are YFP+ but PROX1-LYVE1-PDPN-VWF+EPHB4+. Scale bar: $50 \mu \mathrm{m}$; applies to all panels in B.

LECs were reprogrammed from blood endothelial cells in vessels exposed to flowing blood, but only a small number of such cells could be identified using this Prox $1^{\text {CreERT2 }}$ allele.

The small number of $\mathrm{YFP}^{+}$endothelial cells detected in the mesenteric SVs of Slp76-/-;Prox1 ${ }^{\text {CreERT2}}$;Rosa26RYFP could indicate that most LECs are simply replaced by blood endothelial cells, or it could reflect inefficient labeling due to reduced levels of Prox1 expression in postnatal animals compared with embryos (25) and/ or low levels of CreERT2 expressed behind Prox1 using an IRES in the knock-in Prox $1^{\text {CreERT2 }}$ allele. To distinguish between these possi- 
bilities, we repeated this experiment using a recently described BAC Prox $1^{\text {CreERT2 }}$ transgene that labels postnatal LECs highly efficiently (26). In contrast to lineage tracing studies using the Prox $1^{\text {CreERT2 }}$ knock-in animals, a single tamoxifen injection at P14 resulted in virtually $100 \%$ labeling of LECs in Prox $1^{\text {CreERT2 }}$;Rosa26RYFP BAC transgenic mice, although sparse labeling of venous endothelial cells could also be detected with this regimen (Figure 4B). Analysis of Slp76-1-;Prox $1^{\text {CreERT2; }}$ Rosa26RYFP BAC transgenic mice revealed that in approximately half of SVs, virtually all endothelial cells were $\mathrm{YFP}^{+}$, indicating that these vessels were of lymphatic origin (Figure 4B). The $\mathrm{YFP}^{+}$endothelium of these vessels was uniformly PROX1-LYVE1- ${ }^{-} W^{-} F^{+}$EPHB4 ${ }^{+}$, consistent with a venous blood vessel identity (Figure 4B). These genetic studies demonstrate that the LECs of mesenteric lymphatic vessels of SLP76-deficient mice are neither lost nor replaced during exposure to blood flow, but are instead reprogrammed to venous blood endothelial cells.

Lymphatic endothelial identity is maintained in the presence of blood. Molecular reprogramming of the LECs lining SLP76-deficient mesenteric lymphatic vessels may be a response to factors in the blood that are not present in lymph, to hemodynamic shear forces present in blood vessels but not in lymphatic vessels, or to both. Lethally irradiated wild-type mice that are reconstituted with SLP76-deficient hematopoietic cells develop blood-filled mesenteric lymphatics $(10,27)$, but these mature animals do not undergo subsequent vascular separation and fail to develop the shunts or cardiomegaly observed in $S l p 76^{--}$animals $(10,27)$. Previous angiographic studies of SLP76-deficient mice revealed that paired mesenteric SVs in the anatomic position of the congenital vein and lymphatic are exposed to high levels of blood flow (10). In contrast, angiographic studies performed in SLP76-deficient radiation chimeras revealed minimal blood flow in blood-filled mesenteric lymphatics compared with adjacent veins (Supplemental Videos 1 and 2; supplemental material available online with this article; doi:10.1172/JCI57513DS1), and Doppler ultrasound confirmed that there was minimal blood flow in these vessels (Figure 5, A-D). Unlike the endothelial cells lining Slp $76^{-1-}$ SVs, the endothelial cells lining blood-filled lymphatic vessels in $S l p 76^{-/-}$radiation chimeras exhibited persistent expression of PROX1, PDPN, and LYVE1 and no detectable vWF 16 weeks after reconstitution (Figure 5E). These studies demonstrate that exposure to blood is not sufficient to convert lymphatic vessels to blood vessels in vivo and suggest that fluid flow is necessary.

The fluid shear forces experienced by Slp $76^{-1-}$ mesenteric lymphatics are sufficient to downregulate PROX1 expression in LECs ex vivo. To define a potential link between blood flow and the loss of lymphatic vessel identity in vivo, we calculated the hemodynamic forces in SLP76-deficient mesenteric SVs using high-frequency ultrasound to measure vessel size and blood flow (Figure 6). Two-dimensional (2D) ultrasound of wild-type mesentery revealed a small-caliber artery and a large-caliber vein (Figure 6A). The inability to visualize the wild-type lymphatic vessel was likely due to compression of this very low-pressure vessel by the ultrasound transducer. In contrast, the 3 blood-filled vessels that constitute a typical $\operatorname{Slp} 76^{-/-}$ mesenteric vascular bundle were easily visualized using 2D ultrasound, including a small-caliber artery and two large-caliber SVs (Supplemental Videos 1 and 2, and Figure 6B). Flow profiles in the vessels visualized by $2 \mathrm{D}$ ultrasound were obtained using pulsedwave Doppler ultrasound (Figure 6, C and D). The small-caliber arteries of both wild-type and SLP76-deficient mice displayed high-velocity pulsatile flow in the direction of the intestine (i.e., afferent blood flow) (Figure 6C). The larger-caliber veins in wild- type animals and SVs in SLP76-deficient animals were characterized by non-pulsatile, lower-velocity flow away from the intestine (i.e., efferent blood flow) (Figure 6D). The wall shear stress in each vessel was next calculated using the measured mean flow velocity and vessel diameter, assuming a constant blood viscosity $\eta$ of $7 \mathrm{mPa}^{*} \mathrm{~s}(28-30)$. Shear stresses in wild-type veins and the SVs of SLP76-deficient animals were similar in magnitude, although SVs exhibited greater heterogeneity (Figure 6E and Supplemental Table 1). These studies suggest that mesenteric lymphatic vessels in SLP76-deficient animals become exposed to fluid shear stresses similar to those experienced by mesenteric veins, a result consistent with their molecular venous identity (Figure 3A).

To determine whether fluid shear stress in this range might be sufficient to negatively regulate lymphatic endothelial identity, we subjected primary human LECs to either pulsatile or steady flow with a shear stress of 20 dynes $/ \mathrm{cm}^{2}$. Following 8 hours of shear stress, LECs exhibited a greater than 6-fold reduction in the level of PROX1 mRNA expression compared with static cultured cells (Figure 6F). Exposure to fluid shear forces did not alter expression of the pan-endothelial gene PECAM1 (Figure 6F and Supplemental Figure 1). Expression of the secondary lymphatic markers LYVE1 and $P D P N$ was not significantly reduced in response to shear forces (Figure 6F). Real-time PCR assessment of other endothelial identity markers and known shear-responsive genes revealed little change in the venous markers COUP-TFII and EPHB4, and increases in the arterial markers $H E Y 1, H E Y 2$, and EFNB2, as well as the shear-responsive gene KLF2 (Supplemental Figure 2). The generally arterial shift in gene expression observed in these cells is in contrast to the venous identity observed in vivo and may reflect the difference in the way these forces are exerted, i.e., rapid and full onset in vitro versus slow and gradual onset in vivo. Consistent with the drop in PROX1 mRNA levels, LECs demonstrated dramatically reduced PROX1 protein levels after exposure to shear stress (Figure 6G). Finally, loss of PROX1 mRNA was transient in this system, and levels recovered after 24 hours without shear (Figure $6 \mathrm{H})$. These studies confirm that fluid shear forces like those experienced by lymphatic vessels in postnatal SLP76-deficient animals are sufficient to negatively regulate the expression of the lymphatic endothelial fate regulator PROX1.

\section{Discussion}

Hemodynamic forces have long been postulated to regulate vessel identity, but testing this hypothesis has not been straightforward. The fact that vessel identity is established by a genetic program prior to the presence of significant and diverse hemodynamic forces has made it particularly difficult to discern later contributions of fluid forces on vessel identity, particularly in the case of pathologic flow states such as those in vascular malformations. Most experimental approaches have utilized exogenous, surgical manipulations to dramatically alter blood flow in the developing or mature cardiovascular system to test the effect of hemodynamic forces on vessel identity and cardiovascular organ formation (31-33). While these approaches have provided valuable insights, whether they accurately reflect the more gradual responses that occur in the context of congenital or acquired vascular diseases is unclear. In addition, whether hemodynamic forces can permanently reprogram endothelial and vessel identity is not known. In the present study, we show that postnatal vascular remodeling in SLP76-deficient mice exposes normally formed mesenteric lymphatic vessels to flowing blood that reprograms the LECs lin- 

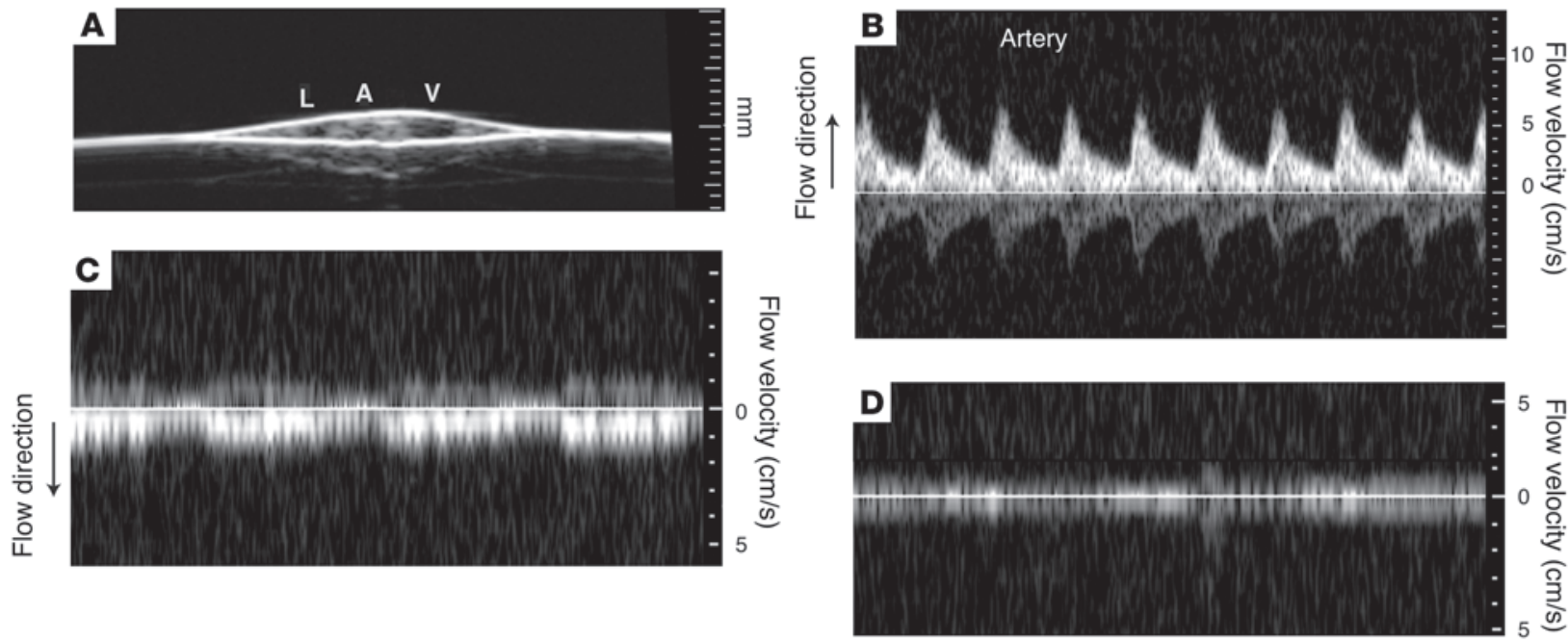

E
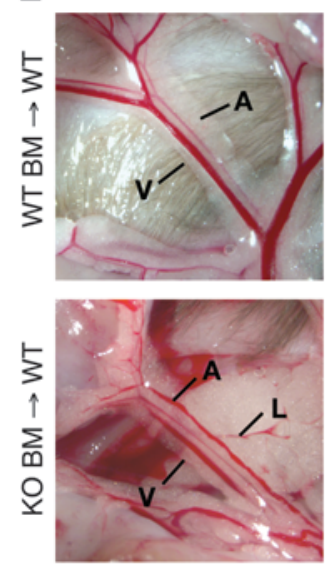

H\&E
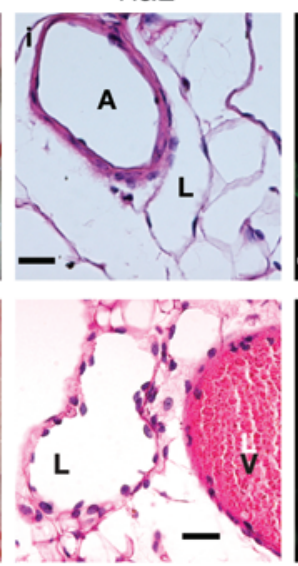

PECAM
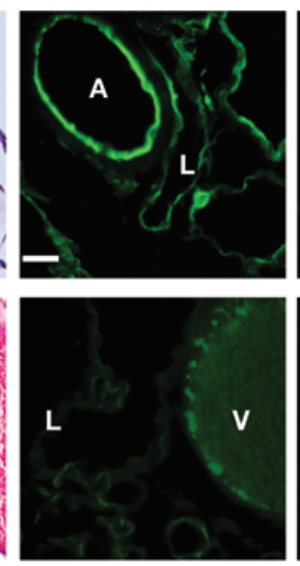

LYVE1/PROX1
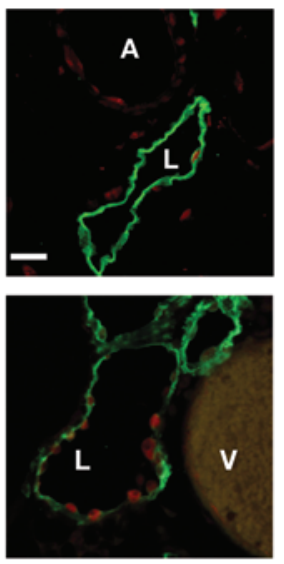

Podoplanin
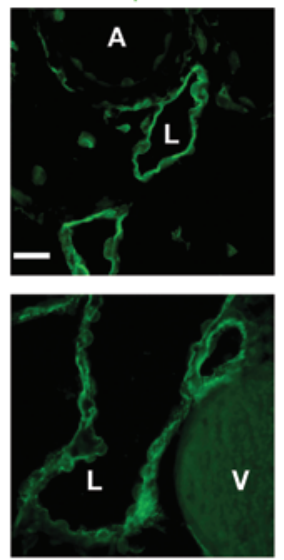

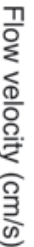

\section{Figure 5}

Lymphatic endothelial identity is maintained in the presence of blood in S/p76 ${ }^{-/-}$radiation chimeras. (A) The mesenteric vessels of SIp76 ${ }^{-/-}$radiation chimeras were visualized using 2D ultrasound. (B-D) Pulsed-wave Doppler signals indicative of blood flow detected in the mesenteric artery, vein, and lymphatic are shown. Note that arterial flow is directed opposite to that of venous flow and that arterial flow is pulsatile (B, corresponding to heart rate), while venous flow is phasic (C, corresponding to respiration). No significant signal in either direction was obtained from the lymphatic (D). (E) Blood-filled mesenteric lymphatics retain lymphatic identity in S/p76- radiation chimeras. Mesenteric vessels in wild-type lethally irradiated mice reconstituted with $S / p 76^{+/+}(+/+)$or SIp76-/- $(-/-)$bone marrow are shown 16 weeks after reconstitution (left). Analysis of serial sections reveals that mesenteric lymphatics in SIp76-- radiation chimeras that are exposed to blood but not flow retain expression of LYVE1, PROX1, and PDPN and do not express vWF (right). Scale bars: $50 \mu \mathrm{m}$.

ing these vessels as blood endothelial cells. Similar changes are observed in LECs exposed to fluid shear ex vivo, suggesting that hemodynamic forces can reprogram lymphatic vessels to blood vessels through transcriptional pathways that establish and maintain endothelial identity.

The key finding in this study is the demonstration that lymphatic vessels exposed to blood flow in vivo after birth are reprogrammed to acquire blood vessel identity. Studies performed more than 30 years ago revealed that endothelial cell turnover in large vessels such as the aorta is low but non-uniform and higher in areas of turbulent blood flow where hemodynamic forces are more varied $(34,35)$. Thus, the onset of significant fluid shear forces in the lymphatic vessels of SLP76-deficient mice could stimulate endothelial turnover and the replacement of LECs by either circu- lating blood endothelial cells or circulating blood endothelial precursor cells believed to contribute to the endothelium of injured or new vessels (36-39). Alternatively, the gradual rise in hemodynamic shear forces may alter the gene expression of the LECs lining these vessels and reprogram them to a blood endothelial identity. Our genetic lineage tracing experiments demonstrate that virtually all of the PROX1-negative endothelial cells that line the SVs of surviving SLP76-deficient animals derive from PROX1-expressing cells. These studies therefore provide definitive evidence of molecular reprogramming of endothelial and vessel identity in response to blood flow in vivo.

Our findings provide strong evidence that hemodynamic forces underlie the reprogramming of lymphatic vessels to blood vessels in response to blood flow. Studies using Vav-Cre;Slp $76^{f /-}$ mice dem- 
A
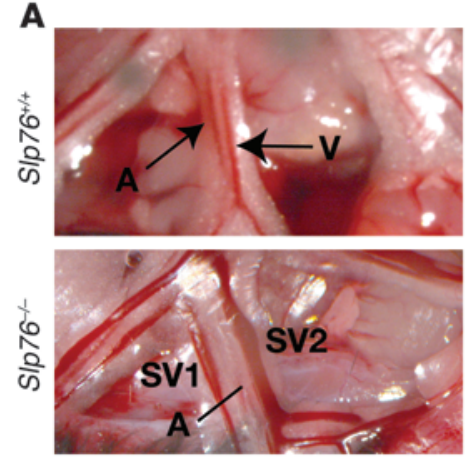

B
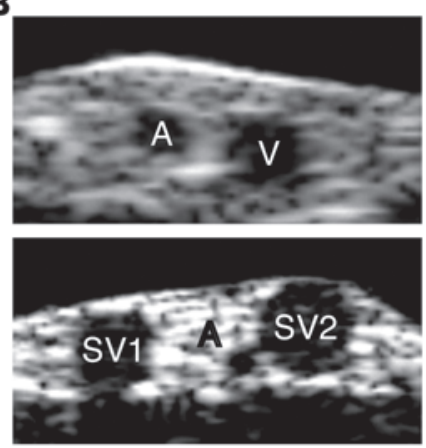

D
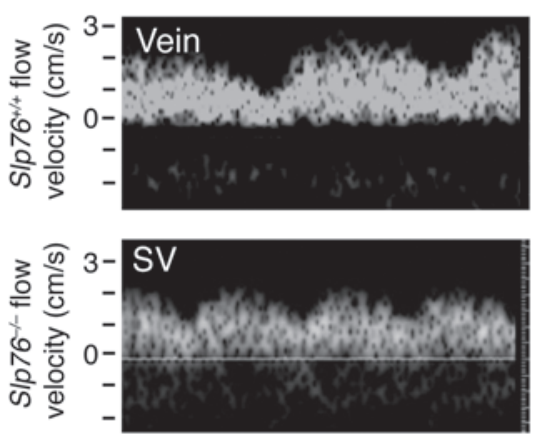

E

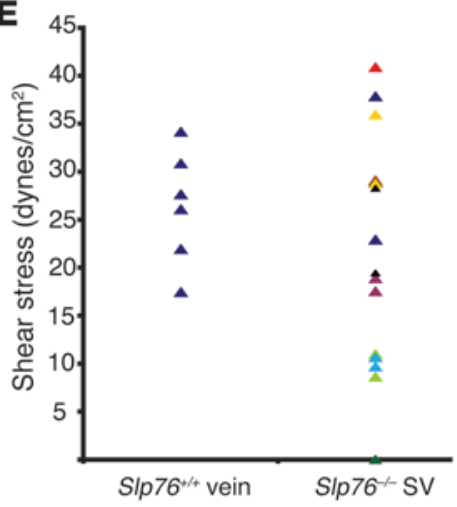

C
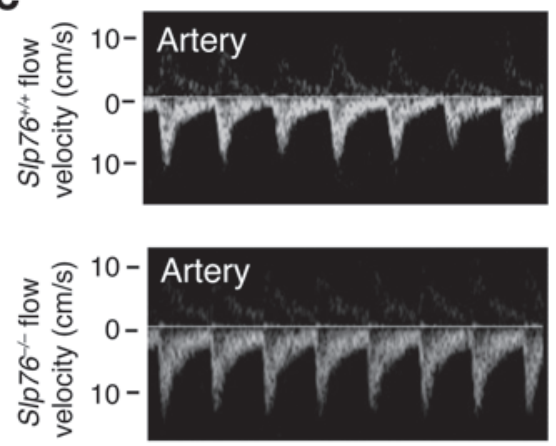

$\mathbf{F}$
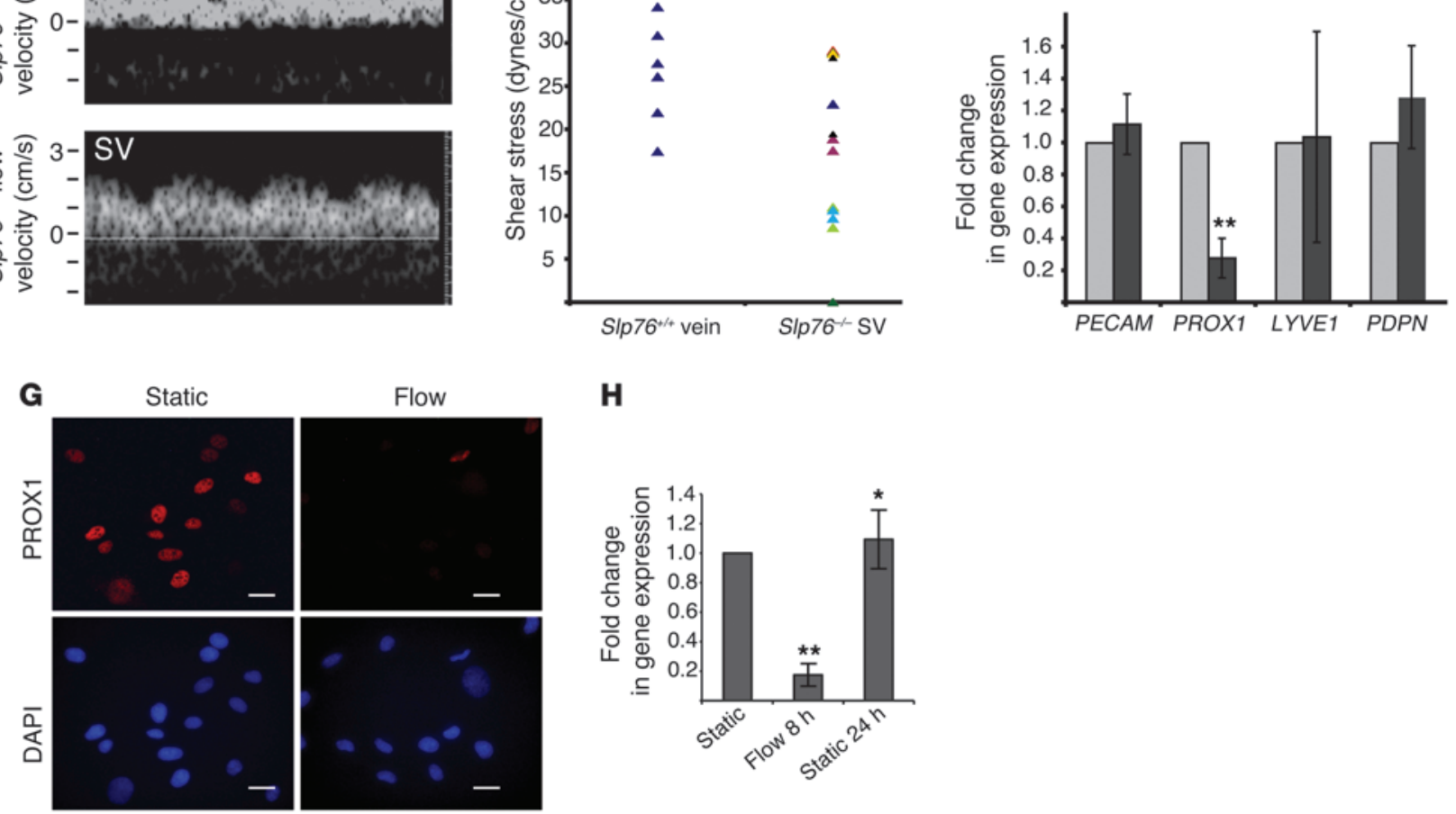

H

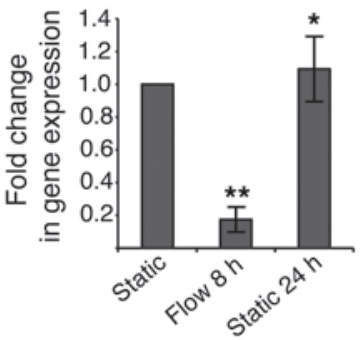

\section{Figure 6}

Fluid shear forces equivalent to those in SIp76--- mesenteric lymphatics drive loss of PROX1 expression in LECs. (A) Representative mesenteric vessels in 12-week-old SIp76 ${ }^{+/+}$and SIp76 ${ }^{-/-}$animals that were studied using Doppler ultrasound. The dilated, blood-containing vein and congenital lymphatic are denoted as SV1 and SV2. (B) The vessels shown in A were visualized using 2D ultrasound. (C and D) The pulsed-wave Doppler signals and measured flow velocity of blood in the vessels indicated in $\mathbf{A}$ and $\mathbf{B}$ are shown. Note the difference in direction of flow between the arteries and veins or efferent SVs. (E) Calculated shear stresses for SIp $76^{+/+}$veins and S/p76 ${ }^{-/-}$SVs. S/p76 ${ }^{-/-}$data points in the same color indicate values of paired SVs from the same mesenteric bundle. (F) LECs exposed to flow downregulate PROX1. LECs were subjected to a shear stress of 20 dynes $/ \mathrm{cm}^{2}$ for 8 hours, and the expression of the indicated mRNAs measured using qPCR. $n=7$. ${ }^{\star \star} P<0.01$. (G) Loss of PROX1 protein in LECs exposed to shear. Anti-PROX1 immunostaining in primary LECs is shown compared with DAPI staining of cell nuclei. (H) Shear-mediated downregulation of $P R O X 1$ is transient in cultured endothelial cells. $P R O X 1$ levels were measured after 8 hours of static culture, 8 hours of flow, or 8 hours of flow followed by 24 hours of static culture. ${ }^{\star} P<0.05,{ }^{\star \star} P<0.01$. Scale bars: $25 \mu \mathrm{m}$.

onstrate that the endothelial and vessel identity changes observed do not reflect an unexpected role for SLP76 in endothelial cells, but instead arise due to changes in the vascular environment that result from blood-lymphatic vascular connections in the intestine. Radiation chimeras reconstituted with SLP76-deficient hematopoietic cells develop blood-filled mesenteric lymphatics but have little or no flow in those vessels, most likely because these mature, irradiated animals do not remodel their vasculature to create arterio-venous-lymphatic shunts like neonatal animals. These animals demonstrate that the formation of blood-lymphatic connections and contact with blood is not sufficient to alter lymphatic endothelial and vessel identity. In contrast, LECs exposed to fluid 
shear forces in the absence of blood ex vivo exhibit rapid downregulation of PROX1. Loss of PROX1 in LECs has been shown to result in the loss of other lymphatic identity markers such as LYVE1 and PDPN $(17,20)$, and genetic deletion of Prox 1 has recently been shown to be sufficient to convert LECs to blood endothelial cells in vivo (18). Thus, our studies support the concept that expression of the PROX1 transcription factor is required to maintain lymphatic endothelial and vessel identity (18) and that loss of PROX1 expression in LECs exposed to fluid shear forces associated with blood flow is a likely mechanism by which lymphatic vessels are reprogrammed to blood vessels in SLP76-deficient mice.

What are the molecular pathways through which hemodynamic forces negatively regulate PROX1 and endothelial lymphatic identity? We found that HEY1 and HEY2 are strongly upregulated in coordination with downregulation of PROX1 in LECs exposed to fluid shear forces (Supplemental Figure 2), and HEY $1 / 2$ or NOTCH expression was recently demonstrated to negatively regulate lymphatic endothelial identity in cultured LECs by reducing expression of PROX1 (40). To test the role of HEY1/2 expression and loss of lymphatic endothelial identity in SLP76-deficient mice in vivo, we have examined Slp76 $6^{-/-} ; H e y 2^{\text {LacZ/+ }}$ mice, in which LacZ is expressed in place of Hey2 (41). However, we did not detect LacZ expression in the endothelium of mesenteric arteries or SVs, suggesting that the NOTCH/HEY signaling is not the basis for this endothelial reprogramming event in vivo. It is possible that flow-dependent activation of HEY $1 / 2$ signaling plays a transient role in this process that was not detected by these studies, but it seems more likely that ex vivo studies of cultured endothelial cells performed over hours do not fully model molecular changes that take place over weeks in vivo. Flow chamber experiments have identified large numbers of endothelial genes that are upregulated and downregulated by fluid flow, including many involved in endothelial identity $(31,42,43)$, but there is no definitive means of testing whether and to what extent these genes mediate endothelial responses to hemodynamic forces in vivo. Transiently blocking blood flow in very young embryos can distinguish between programmed endothelial identity gene expression and gene expression driven by fluid forces $(44,45)$, but this approach is not feasible when hemodynamic changes arise more gradually, as they do in patients with congenital vascular and cardiac defects. Thus, the identity of the molecular signals that downregulate PROX1 expression in response to fluid flow in vivo is not yet defined.

An important implication of this study is that vessel identity remains plastic after vascular development is complete and may be radically altered by hemodynamic forces later in life. The healthy mature vasculature is thought to be very quiescent, but many congenital and acquired human cardiovascular diseases are associated with persistent changes in blood flow and fluid shear forces, e.g., the left-to-right shunting of blood to the low-pressure pulmonary vasculature from the high-pressure arterial system in congenital heart disease. Molecular changes in endothelial and vessel identity are very likely to accompany the hemodynamic alterations in these diseases. Defining these molecular changes is expected to provide new insight into the pathogenesis and treatment of human cardiovascular diseases such as vascular malformations.

\section{Methods}

Histology and immunohistochemistry. Intestine and mesentery were dissected from murine neonates and adults and fixed in $4 \%$ paraformaldehyde for 24-48 hours, dehydrated in $100 \%$ ethanol, and embedded in paraffin. Serial $8-\mu \mathrm{m}$-thick sections were subjected to hematoxylin and eosin and/or immunohistochemical staining as detailed by the University of Pennsylvania Molecular Cardiology Research Center Histology and Gene Expression Core (http://www.med.upenn.edu/mcrc/histology_core/). Antibodies used were MEC13.3 rat anti-mouse CD31 (BD Biosciences - Pharmingen) at 1:500, rabbit anti-LYVE1 at 1:1,000, rabbit anti-Prox1 (Abcam) at 1:100, anti-CX40 (Alpha Diagnostics) at 1:100, and antiEPHB4 (Cell Sciences) at 1:50.

Injection of biotinylated lectin. Intracardiac injection of $50 \mu \mathrm{g}$ of biotinylated Lycopersicon esculentum (tomato) lectin (Vector Laboratories) was performed in neonatal $\operatorname{Sl} 76^{-1-}$ mice and wild-type littermate controls. Co-injection with FITC-dextran (Sigma-Aldrich) was used to determine successful perfusion. Biotinylated tomato lectin (300 $\mu \mathrm{g}$; Vector Laboratories) was injected intravenously into 10 - to 12 -week-old $\operatorname{Slp} 76^{-1-}$ mice and wild-type littermate controls via tail vein. The mice were then euthanized and fixed by perfusion with $4 \%$ paraformaldehyde. Tissues were then processed for immunohistochemical analysis as described above.

Hemodynamic studies. A Visual Sonics Vevo 770 Imaging System with single-element mechanical transducers was used to image the mesenteric vascular structures and blood flow spectra. The center frequency was set at $40 \mathrm{MHz}$ (for lateral and axial resolutions of 68 and $38 \mathrm{~nm}$, respectively). Twelve-week-old mice were anesthetized with Avertin, and their intestine and mesentery were dissected and exposed. Body temperature was monitored via a rectal thermometer and maintained between $36^{\circ} \mathrm{C}$ and $37^{\circ} \mathrm{C}$ using a heating pad and lamp, and heart rate and electrocardiogram were also continuously monitored. To eliminate interference from structures underlying the mesentery, the mesenteric and intestinal areas of interest were spread on a slide glass. A thin layer of prewarmed ultrasound gel was applied to the slide glass and covered with a plastic membrane before tissue exposure. A second membrane was placed on the tissue surface to keep it clean for later dissection. Another layer of prewarmed thick ultrasound gel was placed on this membrane to provide a coupling medium for the transducer. Two mesenteric vascular bundles from each mouse were selected for imaging. Cross-sectional images of the vessels were recorded and the lumen diameters measured by $2 \mathrm{D}$ ultrasonography. The transducer was then rotated $90^{\circ}$ to obtain a longitudinal view of the vessels. For Doppler spectral analysis, the transducer was adjusted for an angle of insonation less than $60^{\circ}$. The mean blood flow velocities were measured and used to calculate wall shear stress for the vessels of interest.

The wall shear stress in each vessel was next calculated using the measured mean flow velocity $V_{\text {mean }}$ and vessel diameter $I D$, assuming a constant blood viscosity $\eta$ of $7 \mathrm{mPa}^{*} \mathrm{~s}(28-30): \tau$ (shear stress) $=\gamma \cdot \eta=\left(4 V_{\text {mean }} \eta\right) / I D$. Although the wall shear rate $\gamma$ was not directly measured, it was calculated from the measured parameters by using Poiseuille's parabolic model of velocity distribution.

LEC isolation. Human microvascular LECs were harvested according to previously published methods $(46,47)$. In brief, microvascular cells were isolated from human foreskins, and endothelial cell colonies were allowed to expand on gelatin-coated tissue culture dishes in EGM-MV growth medium (Lonza). At confluence, cells were detached using Cell Dissociation Solution (Sigma-Aldrich) and trypsin (Lonza). Immunomagnetic purification was performed by incubating the resuspended cells with monoclonal mouse anti-human podoplanin antibody (clone $18 \mathrm{H} 5$, Research Diagnostics Inc.) for 30 minutes at $4^{\circ} \mathrm{C}$, followed by incubation with pan-mouse IgG magnetic beads (Invitrogen) for 15 minutes at $4^{\circ} \mathrm{C}$. LECs attached to beads were magnetically separated and plated on gelatin-coated dishes. LECs were subsequently cultured and passaged using EGM-2 medium supplemented with VEGF or EGM2-MV medium (Lonza). Purity of greater than 95\% was verified using flow cytometry for CD31 and podoplanin. 
Cell culture under shear stress. Passage 4 LECs were cultured to confluence on gelatin-coated glass slides for use in a parallel plate flow chamber. Pulsatile flow with shear stress of 20 dynes $/ \mathrm{cm}^{2}$ was achieved using a Masterflex peristaltic pump and a parallel plate flow chamber provided by J.A. Frangos (CytoDyne, San Diego, California, USA). Non-pulsatile flow with shear stress of 20 dynes $/ \mathrm{cm}^{2}$ was achieved using a 6-slide parallel plate flow chamber designed by Flexcell International Corp. The medium reservoir was maintained at $37^{\circ} \mathrm{C}$ using an incubator or water bath. Cells were incubated in the flow chamber for 8 hours. Static control cells were fed with fresh medium at the beginning of the experiment and maintained in the incubator for the duration of the experiment.

Real-time PCR analysis. Total RNA from LECs (primary isolation described above) and human umbilical vein endothelial cells (Lonza) was isolated using an RNeasy Mini Kit (QIAGEN) according to the manufacturer's protocols. For reverse transcriptase reactions, $1 \mu \mathrm{g}$ of total RNA and 100 ng of random hexamers was used to generate cDNA with the First-Strand cDNA Synthesis Kit (Invitrogen) according to the manufacturer's protocols. Real-time semi-quantitative PCR and relative quantitation normalized to GAPDH was performed using SYBR Green Master Mix reagent (Applied Biosystems) on the ABI Prism 7900HT Sequence Detection System (Applied Biosystems). Real-time PCR primers were designed and obtained from IDT. Primer sequences are listed in Supplemental Methods.

Immunocytochemistry. Cells were fixed in $4 \%$ paraformaldehyde for 15 minutes at room temperature and permeabilized with $0.2 \%$ Triton X-100/PBS. Samples were blocked with $2 \%$ bovine serum albumin in PBS and stained with rabbit anti-Prox 1 antibody (Abcam), followed by Alexa Fluor 488 secondary antibody (Molecular Probes, Invitrogen), and mounted with DAPI-containing Vectashield mounting medium (Vector Laboratories) to visualize cell nuclei.

1. Friedlander RM. Clinical practice. Arteriovenous malformations of the brain. N Engl J Med. 2007; 356(26):2704-2712.

2. Peery WH. Clinical spectrum of hereditary hemorrhagic telangiectasia (Osler-Weber-Rendu disease). Am J Med. 1987;82(5):989-997.

3. Garzon MC, Huang JT, Enjolras O, Frieden IJ. Vascular malformations: Part I. J Am Acad Dermatol. 2007;56(3):353-370.

4. Brouillard P, Vikkula M. Genetic causes of vascular malformations. Hum Mol Genet. 2007; 16(spec no. 2):R140-R149.

5. Oda T, et al. Mutations in the human Jagged 1 gene are responsible for Alagille syndrome. Nat Genet. 1997;16(3):235-242.

6. McAllister KA, et al. Endoglin, a TGF-beta binding protein of endothelial cells, is the gene for hereditary haemorrhagic telangiectasia type 1. Nat Genet. 1994;8(4):345-351.

7. Sahoo T, et al. Mutations in the gene encoding KRIT1, a Krev-1/rap1a binding protein, cause cerebral cavernous malformations (CCM1). Hum Mol Genet. 1999;8(12):2325-2333.

8. McCright B, Lozier J, Gridley T. A mouse model of Alagille syndrome: Notch2 as a genetic modifier of Jag1 haploinsufficiency. Development. 2002; 129(4):1075-1082.

9. Bourdeau A, Dumont DJ, Letarte M. A murine model of hereditary hemorrhagic telangiectasia. J Clin Invest. 1999;104(10):1343-1351.

10. Abtahian F, et al. Regulation of blood and lymphatic vascular separation by signaling proteins SLP-76 and Syk. Science. 2003;299(5604):247-251.

11. Bertozzi CC, et al. Platelets regulate lymphatic vascular development through CLEC-2-SLP-76 signaling. Blood. 2010;116(4):661-670.

12. Turner $M$, et al. Perinatal lethality and blocked B-cell development in mice lacking the tyrosine kinase Syk. Nature. 1995;378(6554):298-302.

13. Clements JL, et al. Fetal hemorrhage and platelet dysfunction in SLP-76-deficient mice. J Clin Invest.
Genetic reconstitution with SLP76-deficient bone marrow and angiography studies. Wild-type mice were lethally irradiated and reconstituted with SLP76deficient bone marrow according to published methods $(10,27)$. Recipient mice were sacrificed for study $8-10$ weeks after transplantation. FITCdextran angiography with real-time video microscopy was performed on anesthetized animals as previously described (10). Tissues were harvested, fixed, and processed for immunohistochemical analysis.

Statistics. $P$ values were calculated using an unpaired, 1-tailed Student's $t$ test. A $P$ value less than 0.05 was considered significant. All bar graphs and error bars represent mean values and SEM.

Study approval. Animal studies were approved by the Institutional Animal Care and Use Committee at the University of Pennsylvania, Philadelphia, Pennsylvania, USA.

\section{Acknowledgments}

This work was supported by an American Heart Association (AHA) award (Scientist Development Award 0730286N to S.J. Stachelek), Cancer Research UK (to T. Makinen), and the National Heart, Lung, and Blood Institute (NHLBI; HL073402 to G. Oliver and HL072798 to M.L. Kahn).

Received for publication February 20, 2012, and accepted in revised form April 5, 2012.

Address correspondence to: Mark L. Kahn, University of Pennsylvania, 952 BRB II/III, 421 Curie Blvd., Philadelphia, Pennsylvania 19104, USA. Phone: 215.898.9007; Fax: 215.573.2094; E-mail: markkahn@mail.med.upenn.edu.
1999;103(1):19-25.

14. Sabin FR. On the origin of the lymphatic system from the veins, and the development of the lymph hearts and thoracic duct in the pig. Am J Anat. 1902;1(3):367-389.

15. Srinivasan RS, et al. Lineage tracing demonstrates the venous origin of the mammalian lymphatic vasculature. Genes Dev. 2007;21(19):2422-2432.

16. Kim KE, Sung HK, Koh GY. Lymphatic development in mouse small intestine. Dev Dyn. 2007; 236(7):2020-2025.

17. Wigle JT, et al. An essential role for Prox 1 in the induction of the lymphatic endothelial cell phenotype. Embo J. 2002;21(7):1505-1513.

18. Johnson NC, et al. Lymphatic endothelial cell identity is reversible and its maintenance requires Prox 1 activity. Genes Dev. 2008;22(23):3282-3291.

19. Kriehuber $E$, et al. Isolation and characterization of dermal lymphatic and blood endothelial cells reveal stable and functionally specialized cell lineages. J Exp Med. 2001;194(6):797-808.

20. Petrova TV, et al. Lymphatic endothelial reprogramming of vascular endothelial cells by the Prox1 homeobox transcription factor. EMBO J. 2002; 21(17):4593-4599.

21. Banerji S, et al. LYVE-1, a new homologue of the CD44 glycoprotein, is a lymph-specific receptor for hyaluronan. J Cell Biol. 1999;144(4):789-801.

22. Nelson GM, Padera TP, Garkavtsev I, Shioda T, Jain RK. Differential gene expression of primary cultured lymphatic and blood vascular endothelial cells. Neoplasia. 2007;9(12):1038-1045.

23. Jiang $S$, et al. Hematopoietic stem cells contribute to lymphatic endothelium. PLoS One. 2008;3(11):e3812.

24. Stadtfeld M, Graf T. Assessing the role of hematopoietic plasticity for endothelial and hepatocyte development by non-invasive lineage tracing. Development. 2005;132(1):203-213.

25. Bazigou E, et al. Integrin-alpha 9 is required for fibronectin matrix assembly during lymphatic valve morphogenesis. Dev Cell. 2009;17(2):175-186.
26. Bazigou E, et al. Genes regulating lymphangiogenesis control venous valve formation and maintenance in mice. J Clin Invest. 2011;121(8):2984-2992.

27. Abtahian F, et al. Evidence for the requirement of ITAM domains but not SLP-76/Gads interaction for integrin signaling in hematopoietic cells. Mol Cell Biol. 2006;26(18):6936-6949.

28 . Vogel J, et al. Transgenic mice overexpressing erythropoietin adapt to excessive erythrocytosis by regulating blood viscosity. Blood. 2003;102(6):2278-2284.

29. Gnasso A, et al. Association between intima-media thickness and wall shear stress in common carotid arteries in healthy male subjects. Circulation. 1996;94(12):3257-3262.

30. Irace C, Cortese C, Fiaschi E, Carallo C, Farinaro E, Gnasso A. Wall shear stress is associated with intima-media thickness and carotid atherosclerosis in subjects at low coronary heart disease risk. Stroke. 2004;35(2):464-468.

31. Buschmann I, et al. Pulsatile shear and Gja5 modulate arterial identity and remodeling events during flow-driven arteriogenesis. Development. 2010; 137(13):2187-2196.

32. le Noble F, et al. Flow regulates arterial-venous differentiation in the chick embryo yolk sac. Development. 2004;131(2):361-375.

33 . Kwei S, et al. Early adaptive responses of the vascular wall during venous arterialization in mice. $\mathrm{AmJ}$ Pathol. 2004;164(1):81-89.

34. Caplan BA, Schwartz CJ. Increased endothelial cell turnover in areas of in vivo Evans Blue uptake in the pig aorta. Atherosclerosis. 1973;17(3):401-417.

35. Schwartz SM, Benditt EP. Clustering of replicating cells in aortic endothelium. Proc Natl Acad Sci US A. 1976;73(2):651-653.

36. Walter DH, et al. Statin therapy accelerates reendothelialization: a novel effect involving mobilization and incorporation of bone marrow-derived endothelial progenitor cells. Circulation. 2002; 105(25):3017-3024

37. Iwakura A, et al. Estrogen-mediated, endothelial 
nitric oxide synthase-dependent mobilization of bone marrow-derived endothelial progenitor cells contributes to reendothelialization after arterial injury. Circulation. 2003;108(25):3115-3121.

38. Ii M, et al. Endothelial progenitor thrombospondin-1 mediates diabetes-induced delay in reendothelialization following arterial injury. Circ Res. 2006;98(5):697-704.

39. Sahara M, et al. Comparison of various bone marrow fractions in the ability to participate in vascular remodeling after mechanical injury. Stem Cells. 2005;23(7):874-878.

40. Kang J, et al. An exquisite cross-control mechanism among endothelial cell fate regulators directs the plasticity and heterogeneity of lymphatic endothelial cells. Blood. 2010;116(1):140-150.

41. Fischer A, Schumacher N, Maier M, Sendtner M, Gessler M. The Notch target genes Hey1 and Hey2 are required for embryonic vascular development. Genes Dev. 2004;18(8):901-911.

42. Jones EA, le Noble F, Eichmann A. What determines blood vessel structure? Genetic prespecification vs. hemodynamics. Physiology (Bethesda). 2006;21:388-395.

43. Parmar KM, et al. Integration of flow-dependent endothelial phenotypes by Kruppel-like factor 2 . J Clin Invest. 2006;116(1):49-58.

44. Jones EA, Yuan L, Breant C, Watts RJ, Eichmann A.
Separating genetic and hemodynamic defects in neuropilin 1 knockout embryos. Development. 2008;135(14):2479-2488.

45. Nicoli S, Standley C, Walker P, Hurlstone A, Fogarty KE, Lawson ND. MicroRNA-mediated integration of haemodynamics and Vegf signalling during angiogenesis. Nature. 2010;464(7292):1196-1200.

46. Veikkola $T$, et al. Intrinsic versus microenvironmental regulation of lymphatic endothelial cell phenotype and function. FASEBJ. 2003;17(14):2006-2013.

47. Richard L, Velasco P, Detmar M. A simple immunomagnetic protocol for the selective isolation and long-term culture of human dermal microvascular endothelial cells. Exp Cell Res. 1998;240(1):1-6. 